\title{
Effect of LED Illumination on the Biological Habits of the Adults of Ectropis grisescens
}

\author{
Li Qiao, ${ }^{1,2}$, Quan Zhang1, Shubao Geng ${ }^{1,2}$, Fangmei Zhang,, Junhua Chen ${ }^{1,2}$ \\ and Shibao Guo ${ }^{1,2, *}$ \\ ${ }^{1}$ Xinyang Agriculture and Forestry University, Xinyang 464000, China \\ ${ }^{2}$ Henan Provincial South Henan Crop Pest Green Prevention and Control \\ Academician Workstation, Xinyang, 464000, China
}

\begin{abstract}
A B S T R A C T
The effects of illumination of four LED wavelengths $(440 \sim 450 \mathrm{~nm}, 460 \sim 465 \mathrm{~nm}, 600 \sim 605 \mathrm{~nm}$ and $490 \sim 495 \mathrm{~nm}$ ) on egg quantity, oviposition rate and adult longevity of Ectropis grisescens Warren were investigated in laboratory. The results showed that the total egg quantity, oviposition rate and adult longevity were all affected. The egg number every female adult of $E$. grisescens Warren was reduced $37.36 \%, 26.17 \%, 41.88 \%$ and $35.11 \%$ contrast to the control. The average oviposition rate reduced $16.61 \%, 11.44 \%, 11.81 \%$ and $8.86 \%$ respectively with the supply of four wave lengths at night. Meanwhile, the pre-oviposition period and oviposition period were affected by the four illumination deals of wave lengths. The longevity of male and female adult was decreased, and the male adult was longer than that of female adult under each treatment of E. grisescens Warren. The eclosion rate of four deals were affected respectively. The purpose was screen out the best spectrum possible by study the effect of spectral treatment on biological habit of $E$. grisescens Warren. The results provide a scientific basis for the use of phototaxis to the integrated management of E. grisescens Warren.
\end{abstract}

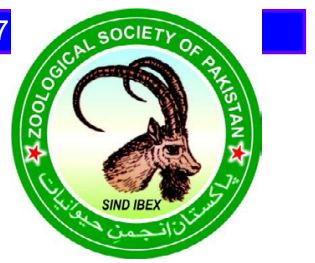

Article Information
Received 01 June 2020
Revised 30 July 2020
Accepted 18 April 2021
Available online 18 May 2021
(early access)
Published 16 November 2021
Authors' Contribution
SG designed the study. LQ wrote the
paper and analyzed the data. SG and
QZ collected the data. FZ provided
guidance and ideas. JC provided the
material support.
Key words
Spectrum, Ectropis grisescens Warren,
Egg quantity, Oviposition rate, Adult
longevity.

\section{INTRODUCTION}

E ctropis grisescens Warren is one of the most serious pests in tea garden, belongs to the Lepidoptera, Geometridae, which seriously affects the quality and yield of tea leaves (Qiao et al., 2020; Zhang et al., 2019; Wang et al., 2018; Ma et al., 2016). The frequent and large amount use of pesticides in production not only causes drug resistance, but also causes pesticide residues, which affects the quality of tea (Zhang et al., 2019; Luo et al., 2018), therefore, it is of great significance to find safe and effective control methods for tea production.

Light trapping is an environmental and effective prevention for control measures. Lighting can disturb the normal growth rhythm of insects, interfere with their mating, oviposition, normal growth and development, or use insect's response to a certain light source, drive them out of the tea garden, reduce the amount of eggs laid in the field, reduce the cost of prevention, thus providing a new idea for pest control (Wang et al., 2020; Liu et al., 2019; Sang et al., 2018). It is reported that insects have strong tendency towards short-wave light or long-wave light (Duan et al., 2009, 2010; Jiang et al., 2008). The test results showed that 505,540 and $589 \mathrm{~nm}$ of illumination

\footnotetext{
* Corresponding author: sbgu0510@163.com 0030-9923/2022/0001-0071\$9.00/0

Copyright 2022 Zoological Society of Pakistan
}

could prolong the oviposition period of the Spodoptera exigua (Hubner), and 505 and $590 \mathrm{~nm}$ illumination could also prolong the early oviposition period of the Plutella xylostella (Linnaeus) and shorten their oviposition period, and 505 and $590 \mathrm{~nm}$ illumination could also prolong the oviposition period of the Helicoverpa armigera Hubner (Chen et al., 2016; Lin et al., 2007). When the wavelength was $560 \mathrm{~nm}$, the phototaxis of Drosophila melanogaster was strong. The light wavelength of $520 \sim 523 \mathrm{~nm}$ and 465 467 nm could affect the growth, development and reproduction of insects (Tu et al., 2014). Yang and Yang have found that red light had the most obvious effect on larval growth, and blue light had the most obvious effect on pupal and eclosion stage (Yang and Yang, 2014). The LED dual-spectrum solar insecticidal lamp composed of violet light and blue light has the most trapping capacity to the Ectropis grisescens in the $380 \sim 400 \mathrm{~nm}$ band (Tu et al., 2019). Some studies have also shown that the spectrum range of 350, 348 and $452 \mathrm{~nm}$ is the best for the trapping and killing Ectropis grisescens (Wang et al., 2018). In recent years, there have been many reports about the biological habit and the regularity of outbreak of Ectropis grisescens, and some reports are about the phototaxis and sexual attractants, but there are no reports about the influence of LED spectrum on the growth of adult Ectropis grisescens.

In this study, lights of different wavelength were used to test the biological characteristics of Ectropis grisescens 
under treatment of blue light at $440 \sim 450 \mathrm{~nm}, 460 \sim 465$ $\mathrm{nm}$, orange light at $600 \sim 605 \mathrm{~nm}$, and ice indigo light at $490 \sim 495 \mathrm{~nm}$, a total of 4 different wavelengths, the adult Ectropis grisescens mortality, egg hatching rate, single female oviposition amount and adult Ectropis grisescens life, etc., providing reference for the effective utilization of spectrum in pest control of Ectropis grisescens.

\section{MATERIALS AND METHODS}

\section{Insect source}

The source of Ectropis grisescens was captured from the tea garden $\left(\mathrm{N} 32^{\circ}, \mathrm{W} 114^{\circ}\right)$ in Baimiao Village, Shihegang Township. Then were fed with fresh tea leaves in artificial climate room, feeding environment was at temperature of $22 \sim 26^{\circ} \mathrm{C}$, relative humidity was $60 \sim 70 \%$, photoperiod was 12L: 12D.

\section{Main equipment}

The main equipment used in this study are listed below: RTOP-310Y artificial climate box (Zhejiang Topu Yunnong Science and Technology Co., Ltd.). PM6612 digital photometer (Shenzhen Huayi Science and Technology Co., Ltd.). Insect cage $(50 \mathrm{~cm} \times 50 \mathrm{~cm} \times 60$ $\mathrm{cm}$ ). Insect cage cover (acrylic transparent round tube with 50 mesh white gauze at the top and a petri dish with a diameter of $9 \mathrm{~cm}$ at the bottom, height of $20 \mathrm{~cm}$ ). Light treatment device $(40 \mathrm{~cm} \times 30 \mathrm{~cm} \times 40 \mathrm{~cm}$ by length, width and height; light intensity: 50 100 lx).

\section{Light sources}

The color and wavelength of the light source are blue light at 440 450 nm, 460 465 nm, orange light at 600 605 $\mathrm{nm}$ and ice indigo light at $490 \sim 495 \mathrm{~nm}$, respectively.

\section{Test methods}

After mating the female and male adults of the newlyemerged Ectropis grisescens, placed them in the insect cover, one pair per cover. The treatment group was given $12 \mathrm{~h}$ (7: 00 19: 00) ordinary fluorescent light (150 200 lx) during the day, and $440 \sim 450 \mathrm{~nm}, 460 \sim 465 \mathrm{~nm}, 600 \sim 605 \mathrm{~nm}$ of orange light or 490 495 $\mathrm{nm}$ of ice indigo light at night for $12 \mathrm{~h}$ (19: 00 7: 00 the following day) in the treatment unit. The light conditions of control group were as follows: $12 \mathrm{~h}$ of normal fluorescent light during the day, and $12 \mathrm{~h}$ of darkness at night. Each treatment for 10 pairs of adult Ectropis grisescens, repeat 3 times. Add 10\% honey water to supplement insect nutrition at regular intervals. Observe the number of eggs laid by the female and the life span of the adult until all the adults die. At the same time, during the $3^{\text {rd }}$ day to the $5^{\text {th }}$ day, select 50 eggs randomly on each day, repeated 3 times, calculate the hatchability of eggs. In addition, collect 20 female and 20 male pupae (start to collect from pre-pupal period, then treat them by male and female, respectively), which were treated under light source for $12 \mathrm{~h}$ every night, and under ordinary fluorescent light source for $12 \mathrm{~h}$ during day. Record the number of pupae, repeated 3 times.

\section{Data analysis}

The data was processed using Excel 2010 software and SPSS 16.0 (SPSS Inc., Chicago, IL). F test was performed for the significant difference between treatments, and Tukey's test for multiple analysis.

\section{RESULTS}

\section{Effect of LED lights on egg-laying}

Under the treatment of 4 lights of different wavelengths, the single female oviposition amount of the adult Ectropis grisescens was significantly lower than that of natural light feeding (Table I). Compared with the control group, the total amount of oviposition decreased by $37.62 \%$ (2568) and maximum decrease of single female oviposition was $41.88 \%$. The difference was significant, and other treatment group were significantly different from the control group. The following treatments were 460 465 $\mathrm{nm}$, with significant differences from other treatments; $440 \sim 450 \mathrm{~nm}$ and 600 605 nm were significantly different from other treatments; the total oviposition and single

Table I.- Effects of different wavelength spectra of LED light on oviposition of E. grisescens adult.

\begin{tabular}{|c|c|c|c|c|c|c|}
\hline Treatment & $\begin{array}{c}\text { Total spawned/ } \\
\text { granule }\end{array}$ & $\begin{array}{c}\text { Single female } \\
\text { spawning/granule }\end{array}$ & $\begin{array}{c}\text { Highest egg } \\
\text { production/granule }\end{array}$ & $\begin{array}{c}\text { Spawning } \\
\text { rate }(\%)\end{array}$ & $\begin{array}{c}\text { Preoviposition } \\
\text { period (d) }\end{array}$ & $\begin{array}{l}\text { Spawning } \\
\text { time (d) }\end{array}$ \\
\hline $440 \sim 450 \mathrm{~nm}$ & $4590.00 \pm 155.88^{c}$ & $306.00 \pm 10.39^{c}$ & 589 & 73.73 & $2.93 \pm 0.18^{\mathrm{a}}$ & $3.63 \pm 0.24^{\mathrm{bc}}$ \\
\hline $460 \sim 465 \mathrm{~nm}$ & $5410.00 \pm 177.97^{b}$ & $360.66 \pm 11.86^{\mathrm{b}}$ & 699 & 86.67 & $2.16 \pm 0.23^{\mathrm{b}}$ & $4.03 \pm 0.13^{b}$ \\
\hline $490 \sim 495 \mathrm{~nm}$ & $4259.00 \pm 120.67^{d}$ & $283.93 \pm 8.05^{\mathrm{d}}$ & 390 & 93.00 & $2.76 \pm 0.09^{\mathrm{a}}$ & $3.43 \pm 0.09^{c}$ \\
\hline $600 \sim 605 \mathrm{~nm}$ & $4754.67 \pm 144.64^{c}$ & $316.98 \pm 9.64^{\mathrm{c}}$ & 403 & 95.00 & $1.83 \pm 0.23^{\mathrm{bc}}$ & $4.56 \pm 0.15^{b}$ \\
\hline $\mathrm{CK}$ & $6827.33 \pm 163.54^{\mathrm{a}}$ & $488.49 \pm 10.90^{\mathrm{a}}$ & 780 & 100.00 & $1.59 \pm 0.36^{\mathrm{c}}$ & $6.48 \pm 0.74^{\mathrm{a}}$ \\
\hline
\end{tabular}

Different small letters following the data in a column indicate significant differences among 0.05 treatments, the same letter indicates no significant difference among treatments (the values in the table are mean \pm standard error, the same below). 
female oviposition at $490 \sim 495 \mathrm{~nm}$ were the lowest. The highest oviposition of single female was the control group, and that of other treatments were all decreased, and their oviposition rate were all lower than that of control, with a maximum decrease value of $26.27 \%$, light treatment led to some adults not oviposit. Spectral treatment prolongs the early oviposition period. Except at $600 \sim 605 \mathrm{~nm}$ treatment, there were significant difference between the four kinds of spectral treatment groups and the control group. The oviposition period under four kinds of spectral treatments were shortened obviously, and the longest shortening time was 3.05 days, and the difference was significant.

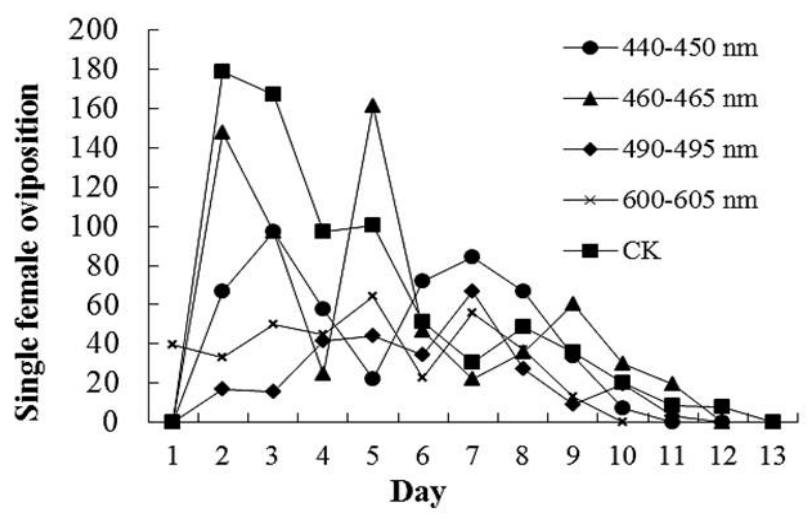

Fig. 1. Effects of LED light of different wavelengths on oviposition of E. grisescens adult.

Four kinds of LED spectra all have a certain influence on the spawning dynamics of the adult Ectropis grisescens (Fig. 1). First, the total amount of each treatment was significantly lower than that of control group. Secondly, the peak time of oviposition was the same as that of control group (460 465 nm) or delayed (440 450 nm and $465 \sim 465 \mathrm{~nm}$ ), appeared on the $3^{\text {rd }}$ and $5^{\text {th }}$ day, respectively. The peak time of treatment at 490 495 nm d 600 605 $\mathrm{nm}$, was not obvious, appeared on the $7^{\text {th }}$ and the $5^{\text {th }}$ day, respectively. The average maximum oviposition of single female was 179 (CK), followed by $161(460 \sim 650 \mathrm{~nm})$, and $98(440 \sim 450 \mathrm{~nm})$. The overall trend showed that the oviposition peak period was during the $2^{\text {nd }}$ day to $7^{\text {th }}$ day, then decreased gradually. The oviposition period of female adults treated at 460 465 nm and 490 495 nm was 11 days, only less than control group (12 days). The oviposition period of female adults treated at $600 \sim 605 \mathrm{~nm}$ was the shortest.

\section{Effect of LED lights on egg incubation rate}

Figure 2 showed the illumination with LED lights of different wavelength treatments have great influence on hatching rate of Ectropis grisescens eggs. The hatching rate of control group was the highest, and significantly different from other treatment groups. The difference between treatments at $460 \sim 465 \mathrm{~nm}, 490 \sim 495 \mathrm{~nm}$ and 600 605 $\mathrm{nm}$ was the same. The difference between treatments at $440 \sim 450 \mathrm{~nm}$ and $600 \sim 605 \mathrm{~nm}$ was significant, and the others have no difference. Compared with the control group, the hatching rate of eggs decreased by $16.61 \%$, $11.44 \%, 11.81 \%$ and $8.86 \%$, respectively.

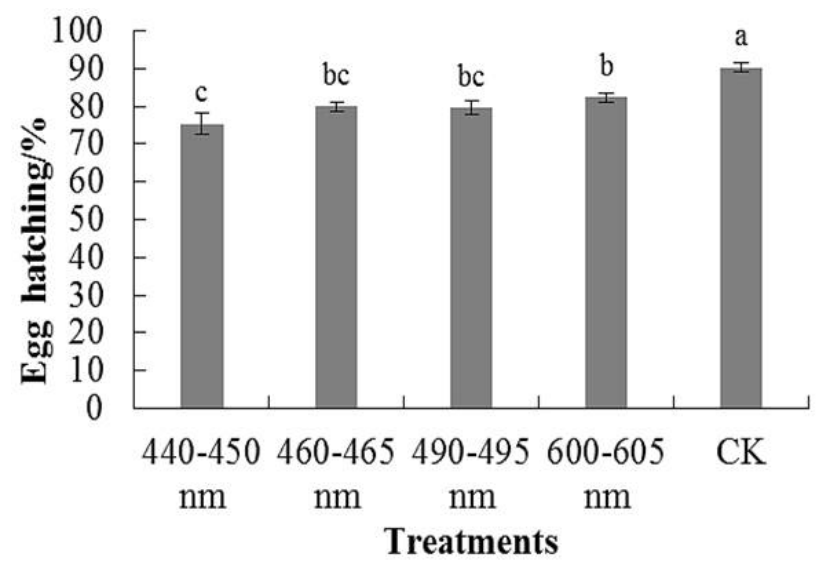

Fig. 2. Effects of LED light of different wavelengths on egg hatching rate of E. grisescens.

Table II.- Effects of LED light of different wavelengths on adult longevity of $E$. grisescens adult.

\begin{tabular}{lcc}
\hline Treatment & Male (d) & Female (d) \\
\hline $440 \sim 450 \mathrm{~nm}$ & $6.83 \pm 0.18^{\mathrm{c}}$ & $6.67 \pm 0.29^{\mathrm{b}}$ \\
$460 \sim 465 \mathrm{~nm}$ & $7.47 \pm 0.27^{\mathrm{bc}}$ & $7.27 \pm 0.18^{\mathrm{a}}$ \\
$490 \sim 495 \mathrm{~nm}$ & $7.86 \pm 0.52^{\mathrm{b}}$ & $6.73 \pm 0.56^{\mathrm{b}}$ \\
$600 \sim 605 \mathrm{~nm}$ & $7.30 \pm 0.10^{\mathrm{bc}}$ & $6.59 \pm 0.25^{\mathrm{b}}$ \\
$\mathrm{CK}$ & $9.16 \pm 0.33^{\mathrm{a}}$ & $7.73 \pm 0.18^{\mathrm{a}}$ \\
\hline
\end{tabular}

Influence of LED lights on the life of adult

As can be seen from Table II, the life span of male and female adult Ectropis grisescens decreased under 4 kinds of spectral treatments, and the difference of male adults between in treatment group and that in control group was significant. Except for treatment of female adults at 460 465 nm with no difference from control group, and the other treatment trends were at the same level. The life span of male adults under each treatment was higher than that of female adults. The life span of female and male adults was reduced by 1.06 days and 2.33 days, respectively under $440 \sim 450 \mathrm{~nm}$ treatments, and reduced by 0.46 days and 1.69 days under $460 \sim 465 \mathrm{~nm}$ treatments, reduced by 1 days and 1.3 days under 490 495 nm treatments, respectively. Female and male adults reduced 
by 1.14 days and 1.86 days under $600 \sim 605 \mathrm{~nm}$ treatments compared with control group. Thus, the life span of female and male adults was decreased in certain degree under 4 kinds of spectral treatments.

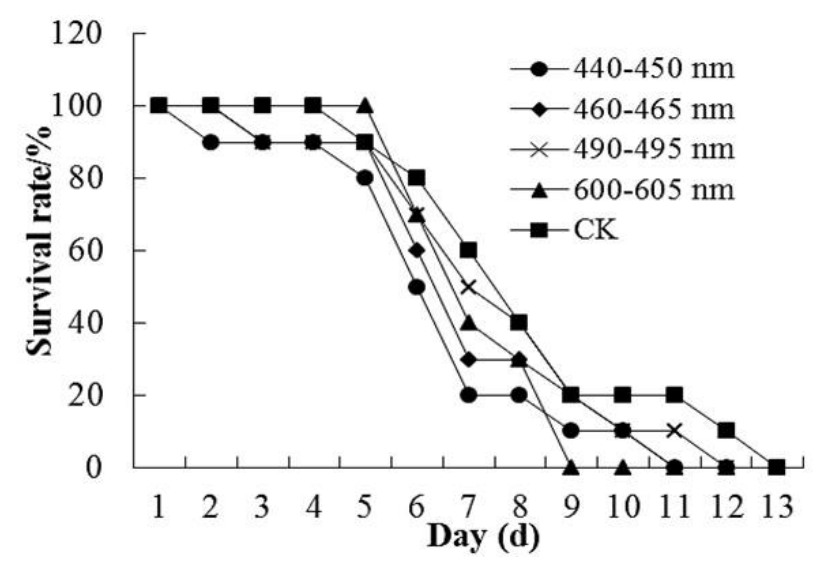

Fig. 3. Effects of LED light of different wavelengths on female adult survival rate of $E$. grisescens adult.

From Figure 3, we can see that spectral treatment has certain effect on the survival rate of female adult, and the survival rate of 6 th day decreased obviously. The life span of the female adult was the longest in control group, followed by 490 495 nm, and the maximum life span of the adult was 11 days, but the maximum life span of the adult was 8 days under $600 \sim 605 \mathrm{~nm}$ treatment and more affected by spectrum. The maximum life span under 440 45 nm and 460 465 nm treatment were both 10 days. The survival rates of all treatments were decreased with time. The survival rate of the adult under four kind spectrums treatments was basically the same as those in control group (Fig. 4). The survival rate of adults under $440 \sim 450 \mathrm{~nm}$ and 600 605 $\mathrm{nm}$ treatment was $13 \mathrm{~d}$, and the life of other treatments was $12 \mathrm{~d}$ as control group. The survival rates of adults in different stages under 4 kinds of spectra were interacted with each other.

\section{Effect of LED lights on eclosion rate of adult}

It can be seen from Figure 5 that all the 4 kinds of spectrum treatment have influence on the eclosion rate of adults, and the control group is the highest, which is significantly different from other treatments. The eclosion rate under 490 495 $\mathrm{nm}$ treatment is the lowest, except the difference is the same as that under $440 \sim 450 \mathrm{~nm}$ treatment is significantly different from other treatment groups. The difference of eclosion rate under the other 3 kinds of spectrum treatment was at the same level.

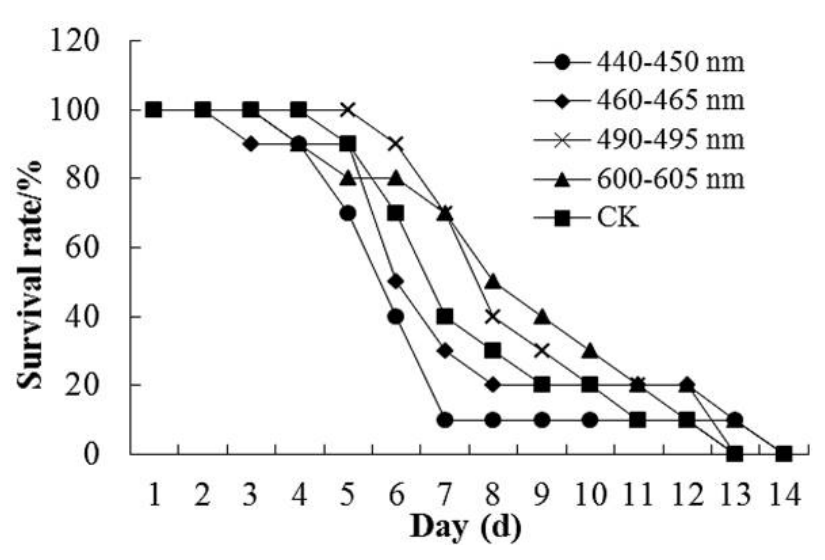

Fig. 4. Effects of LED light of different wavelengths on male adult survival rate of $E$. grisescens adult.

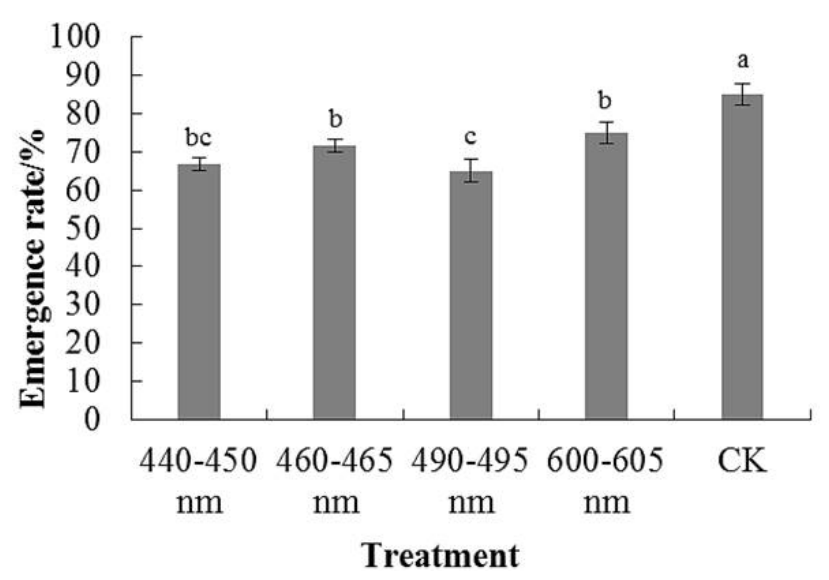

Fig. 5. Effects of LED light of different wavelengths on emergence rate of E. grisescens adult.

\section{DISCUSSION}

Light is an important influencing factor in insect's life and activity. Lights of different colors can cause some changes in insect's taxis behavior and affect the growth and reproduction ability of some insects (Zhang et al., 2018). Under the environment of promoting ecological agriculture and organic planting, using light source to prevent and control pests is the trend of development (Wang et al., 2018).

In this study, under the treatment of 4 spectra at 440 450 nm, 460 465 nm, 490 495 nm and 600 605 $\mathrm{nm}$, the oviposition amount of the Ectropis grisescens decreased by $32.77 \%, 20.76 \%, 37.62 \%$ and $30.36 \%$, respectively. The oviposition rate decreased obviously, the oviposition period was shortened, and the difference was significant from that of control group. In addition, all the four kinds of spectra had an effect on the oviposition 
dynamics of the adults, and the peak time of oviposition was from $2^{\text {nd }}-7^{\text {th }}$ day, and the spawning period was obviously shortened compared with control group. Previous studies on the effect of yellow light on the reproduction of Plutella xylostella showed that the oviposition amount decreased and the oviposition period was shortened (Duan et al., 2007). In this paper, compared with the control group, the hatching rate decreased, which is consistent with the previous studies. In the four spectral treatments, the lifespan of both male and female Ectropis grisescens was reduced. Under the treatment at $440 \sim 450 \mathrm{~nm}$, the lifespan of both male and female Ectropis grisescens reduced by 1.06 days and 2.33 days, respectively, reduced by 0.46 days and 1.69 days under the treatment at $460 \sim 465 \mathrm{~nm}$, reduced by 1.0 days and 1.3 days under the treatment at $490 \sim 495 \mathrm{~nm}$, reduced by 1.14 days $\mathrm{d} 1.86$ days under the treatment at $600 \sim 605 \mathrm{~nm}$, respectively. Thus it can be seen the survival rate of the female was decreasing with time, and the male's survival rate appeared alternately at different stages. At the same time, the spectral treatment had a certain effect on eclosion rate of adult, and eclosion rate was the lowest under treatment at 490 495 nm, except the same difference as treatment at $440 \sim 450 \mathrm{~nm}$, was significantly different from that of other treatments. The results showed that treatment at $490 \sim 495 \mathrm{~nm}$ had a great influence on the eclosion rate of the adults.

In this study, we used insect phototaxis to interfere with their fertility rhythm so as to control their population, and finally reduce the incidence of the next generation of larvae to below the amount which causing economic losses, and improve the yield, quality and ecological environment of tea garden. At present, most of the trapping lamps used in production are mixed continuous light sources, which can achieve better results in pest monitoring and trapping, but also cause some harm to non-target insects such as natural enemies (Tu et al., 2014). Therefore, it is of great practical significance to design and develop special lamps according to the specific sensitive spectrum of pest occurrence.

\section{CONCLUSION}

In conclusion, this study showed that light at $440 \sim 450$ $\mathrm{nm}, 460 \sim 465 \mathrm{~nm}, 600 \sim 605 \mathrm{~nm}$ and 490 495 nm had different effects on the parameters of Ectropis grisescens including oviposition prophase, average oviposition period, single female oviposition rate, hatching rate and life span. Providing light in the tea garden from 7:00 p.m. to 7: $00 \mathrm{a} . \mathrm{m}$. in the next morning before the eclosion of Ectropis grisescens adults could effectively interfere the population development and provide reference for green prevention and control technology of tea garden.

\section{ACKNOWLEDGEMENT}

We acknowledge the financial supports of the national key research and development program (Grant No. 2016YFD0200900) and Young Scientific Research Foundation of Xinyang Agriculture and Forestry University (2018LG003).

\section{Ethical compliance}

There are no researches conducted on animals or humans.

\section{Statement of conflict of interest}

The authors have declared no conflict of interests.

\section{REFERENCES}

Chen, Z., Xu, R., Kuang, R.P. and Sun, R., 2016. Phototactic behaviour of the parasitoid Encarsia formosa (Hymenoptera: Aphelinidae). Biocontr. Sci. Technol., 26: 250-262. https://doi.org/10.1080/ 09583157.2015.1099071

Duan, Y., Wu, R.H., Wu, Y.Q., Jiang, Y.L. and Zhao, M.Q., 2010. Effects of LED illumination on the biology of Plutella xylostella. J. Henan agric. Sci., 1: $80-82$.

Duan, Y., Wu, Y.Q., Jiang, Y.L., Wu, R.H. and Zhao, M.Q., 2009. Effects of LED (light emitting diode) illumination on light adaptation and mating of Helicoverpa armigera. Acta Ecol. Sin., 29: 47274731.

Duan, Y., Wu, Y.Q., Yang, S.F., Wu, R.H. and Zhao, M.Q., 2007. Effects of yellow light on the biology of Plutella xylostella. Pl. Protect., 33: 110-112.

Jiang, Y.L., Duan, Y. and Wu, Y.Q., 2008. Effects of green-yellow light with three different wavelengths on the oviposition biology of Spodoptera exigua (Hübner). Acta Phytophyl. Sin., 35: 473-474.

Lin, M., Yao, B.Y., Zhang, Y.H. and Re, Z.W., 2007. The studies of solar LED lights for killing worms. Renewab. Ener. Resour., 25: 79-80.

Liu, Q., Gao, H.H., Liu, S., Zhai, Y.F., Wei, G.H. and Yu, Y., 2019. A comparative study on the phototaxis behaviors of Drosophila suzukii and D. melanogaster. J. Pl. Protect., 46: 499-500.

Luo, Z.X., Su, L., Li, Z.Q., Liu, Y., Cai, X.M., Bian, L., Xin, Z.J. and Chen, Z.M., 2018. Field application technology of sex pheromone on Ectropis grisescens. J. Tea Sci., 38: 140-145.

Ma, T., Xiao, Q., Yu, Y.G., Wang, C., Zhu, C.Q., Sun, Z.H., Chen, X.Y. and Wen, X.J., 2016. Analysis 
of tea geometrid (Ectropis grisescens) pheromone gland extracts using GC-EAD and $\mathrm{GC} \times \mathrm{GC} /$ TOFMS. J. agric. Fd. Chem., 64: 3161-3166. https://doi.org/10.1021/acs.jafc.6b00339

Qiao, L., Hong, F., Jin, Y.L., Geng, S.B. and Guo, S.B., 2020. Effects on the photoreaction behavior of Ectropis grisescens Warren to different light spectrum. J. Tea Sci., 40: 617-624.

Sang, W., Cai, F.Y., Wang, X.P., Zhang, S., Huang, Q.Y., Zhu, F., Guo, S.H. and Lei, C.L., 2018. Application status and prospects of insect trapping lamp in fields. China Pl. Protect., 38: 26-30. https://doi. org/10.1002/9781119120759.ch1_3

Tu, H.H., Hu, X.X., Mao, Y. and Kuang, X.F., 2019. Development and application of the dual spectrum LED insecticidal lamp for tea garden dedicated. $J$. Anhui Agric. Sci., 47: 114-117.

Tu, X.Y., Chen, Y.S. and Zhi, Y., 2014. Effects of lightemitting diode illumination on insect behavior and biological characters. Pl. Protect., 40: 11-15.

Wang, C.W., Yang, Y.Q., Qian, L.J., Wan, X.C. and Huang, Y.Z., 2018. Trapping effect of different light sources from frequency trembler grid lamp on major insects in tea fields of southern Anhui. $J$. Anhui Agric. Univ., 45: 790-795.

Wang, F.F., Wang, Y., Chen, Y.C., Liu, Y.M., Guo, S.H., Qiu, B.L. and Sang, W., 2020. Phototaxis of
Diaphorina citri Kuwayama (Hemiptera: Liviidae) to LED lights. J. environ. Ent., 42: 187-192.

Wang, Z.B., Ma,T., Mao, T.F., Guo, H.W., Zhou, X.G., Wen, X.J. and Xiao, Q., 2018. Application technology of the sex pheromone of the tea geometrid Ectropis grisescens (Lepidoptera: Geometridae). Int. J. Pest Manage., 64: 372-378. https://doi.org/10.1080/09670874.2018.1447170

Yang, Z.X. and Yang, C.M., 2014. Influence of red, green and blue lights on drosophilas' growth. $J$. Chuxiong Norm. Univ., 29: 68-72.

Zhang, F.M., Liu, S.H., Chen, L.J., Chen, J.H., Yin, J. and Pan, P.L., 2019. Fumigant activity of Chenopodium ambrosioides oil and its effects on acetylcholinesterase and carboxylesterase in Ectropis grisescens. China Pl. Protect., 39: 13-16.

Zhang, J.J., Zhang, X., Du, W.M., Zang, L.S. and Ruan, C.C., 2018. Effects of LED Lights of different wavelengths on the development and reproduction of Trichogramma japonicum. Chin. J. biol. Contr., 34: 204-208.

Zhang, J.X., Sun, Q.Y., Ge, C.M., Ye, T., Zhang, R. and Ding, Y., 2019. Morphological Characteristics and histological structure of the reproductive organs in female adults of Ectropis grisescens Warren (Lepidoptera: Geometridae). J. Tea Sci., 39: 98104. 